\title{
Fishbone Model for Belt Object Deformation
}

\author{
Hidefumi Wakamatsu and Eiji Arai \\ Dept. of Materials and Manufacturing Science, \\ Graduate School of Eng., \\ Osaka University \\ 2-1 Yamadaoka, Suita, Osaka 565-0871, Japan \\ Email: \{wakamatu, arai\}@mapse.eng.osaka-u.ac.jp
}

\begin{abstract}
A modeling method for representing belt object deformation is proposed. Deformation of belt objects such as film circuit boards or flexible circuit boards must be estimated for automatic manipulation and assembly. In this paper, we assume that deformation of an inextensible belt object can be described by the shape of its central axis in a longitudinal direction called "the spine line" and lines with zero curvature called "rib lines". This model is referred to as a "fishbone model" in this paper. First, we describe deformation of a rectangular belt object using differential geometry. Next, we propose the fishbone model considering characteristics of a developable surface, i.e., a surface without expansion or contraction. Then, we formulate potential energy of the object and constraints imposed on it. Finally, we explain a procedure to compute the deformed shape of the object and verify the validity of our proposed method by comparing some computational results with experimental results.
\end{abstract}

\section{INTRODUCTION}

According to downsizing of various electronic devices such as note PCs, mobile phones, digital cameras, and so on, more film circuit boards or flexible circuit boards illustrated in Fig.1 are used instead of conventional hard circuit boards. It is difficult to assemble such flexible boards by a robot because they can be easily deformed during their manipulation process and they must be deformed appropriately in the final state. For example, the flexible circuit board shown in Fig.1-(a) must deform to the objective shape illustrated in Fig.1-(b) to install into the hinge part of a mobile phone. Therefore, analysis and estimation of deformation of film/flexible circuit boards is required.

In solid mechanics, Kirchhoff theory for thin plates and Reissner-Mindlin theory for thick plates have been used[1]. For very thin plates, the inextensional theory was proposed[2]. In this theory, it is assumed that the middle surface of a plate is inextensional, that is, the surface of the plate is developable. Displacement of plates can be calculated using FEM based on these theories. However, the high aspect ratio of thin objects often causes instability in computation of deformed shapes. In computer graphics, a deformable object is represented by a set of particles connected by mechanical elements[3]. Recently, fast algorithms have been introduced to describe linear object deformation using the Cosserat formulation[4]. Cosserat elements possess six degrees of freedom; three for translational displacement and three for rotational displacement. Flexure, torsion, and extension of a linear object can be

\author{
Shinichi Hirai \\ Dept. of Robotics, \\ Ritsumeikan University \\ 1-1-1 Noji Higashi, Kusatsu, Shiga 525-8577, Japan \\ Email: hirai@se.ritsumei.ac.jp
}

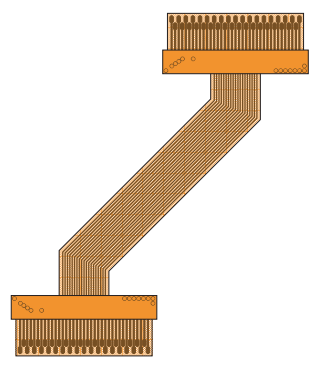

(a) natural shape

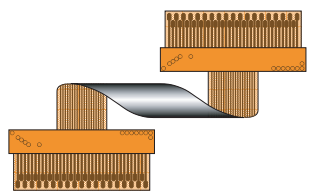

(b) objective shape
Fig. 1. Example of flexible circuit board

described by use of Cosserat elements. In robotics, insertion of a wire into a hole in 2D space has been analyzed using a beam model of the wire to derive a strategy to perform the insertion successfully[5][6]. Kosuge et al. have proposed a control algorithm of dual manipulators handling a flexible sheet metal[7]. Lamiraux et al. have proposed a method of path planning for elastic object manipulation with its deformation to avoid contact with obstacles in a static environment[8]. Dynamic modeling of a flexible object with an arbitrary shape has been proposed to manipulate it without vibration[9]. In differential geometry, curved lines in 2D or 3D space have been studied to describe their shapes mathematically[10]. Moll et al. have proposed a method to compute the stable shape of a linear object under some geometrical constraints quickly based on differential geometry[11]. It can be applied to path planning for flexible wires. We have proposed a modeling method for linear object deformation based on differential geometry and its applications to manipulative operations[12]. In this method, linear object deformation with flexure, torsion, and extension can be described by only four functions. We can simulate various deformation such as torsional buckling, knotted shape, and so on. This method can be applied to a sheet object if the shape of the object is regarded as rectangle, namely, the object has belt-like shape. However, in [12], it is assumed that the shape of cross-section of a linear object is fixed. This assumption is not appropriate to represent 3D shape of a belt object because the shape of its cross-section can change due to deformation.

In this paper, we propose a fishbone model based on differential geometry to represent belt object deformation. In 


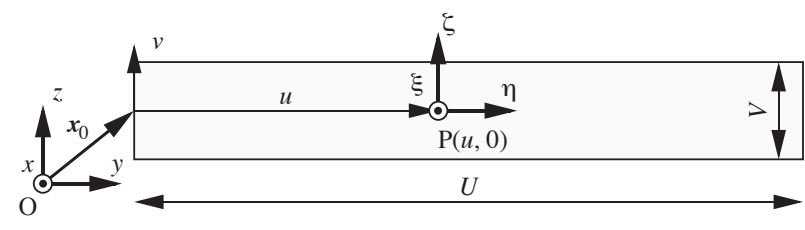

Fig. 2. Coordinates of belt object

this model, deformation of a belt object is represented using its central axis in a longitudinal direction referred to as the spine line and lines with zero curvature referred to as rib lines. The objective of manipulation of a flexible circuit board is to connect its ends to other devices. So, it is important to estimate position and orientation of ends of the board. This implies that we have to estimate more accurately its deformation in a longitudinal direction than that in a transverse direction. The fishbone model is suitable for representation of deformation in a longitudinal direction, that is, deformed shape of the spine line. Moreover, we can estimate belt object deformation if only the flexural rigidity of the object along the spine line is given. It indicates that we can easily identify the actual parameter of the object from experiment. First, we describe deformation of a rectangular belt object using differential geometry. Next, we propose the fishbone model considering characteristics of a developable surface, i.e., a surface without expansion or contraction. After that, we formulate potential energy of the object and constraints imposed on it. Finally, a procedure to compute the deformed shape of the object was explained and some computational results are compared with experimental results.

\section{Modeling of Belt ObJect}

\section{A. Differential Geometry Coordinates}

In this section, we formulate the deformation of a belt object in 3D space. Assumptions in this paper are as follows:

- A belt object has rectangular shape.

- The width of the belt object is sufficiently small compared to its length.

- The object is inextensible. Namely, it can be bent and twisted but cannot be expanded or contracted.

- Both ends of the object cannot be deformed because connectors are attached to the ends.

In this paper, we focus on deformation of the central axis in a longitudinal direction of a belt object and attempt to represent the whole shape of the object using it.

Let $U$ and $V$ be the length and the width of the object, respectively. Let $u$ be the distance from one end of the object along the central axis in its longitudinal direction and let $v$ be the distance from the central axis in a transverse direction of the object. Let $\mathrm{P}(u, v)$ be a point on the object. In order to describe deformation of the central axis of a belt object, the global space coordinate system and the local object coordinate systems at individual points on the object are introduced as shown in Fig.2. Let $\mathrm{O}-x y z$ be the coordinate system fixed in space and $\mathrm{P}-\xi \eta \zeta$ be the coordinate system fixed at an arbitrary point $\mathrm{P}(u, 0)$ on the central axis of the object. Assume that the central axis in a longitudinal direction of the object is parallel to the $y$-axis and the normal vector of any point on the object is parallel to the $x$-axis in its natural state whereby the object has no deformation. Select the direction of coordinates so that the $\xi-, \eta-$, and $\zeta$-axes are parallel to the $x-, y-$, and $z$-axes, respectively, at the natural state. Deformation of the object is then represented by the relationship between the local coordinate system P- $\xi \eta \zeta$ at each point on the object and the global coordinate system O$x y z$. This is referred to as differential geometry coordinate representation. Let us describe the orientation of the local coordinate system with respect to the space coordinate system by use of Eulerian angles, $\phi(u, 0), \theta(u, 0)$, and $\psi(u, 0)$. The rotational transformation from the coordinate system P- $\xi \eta \zeta$ to the coordinate system $\mathrm{O}-x y z$ is expressed by the following rotational matrix:

$$
\begin{aligned}
& A(\phi, \theta, \psi)= \\
& {\left[\begin{array}{c|c|c}
C_{\theta} C_{\phi} C_{\psi}-S_{\phi} S_{\psi} & -C_{\theta} C_{\phi} S_{\psi}-S_{\phi} C_{\psi} & S_{\theta} C_{\phi} \\
C_{\theta} S_{\phi} C_{\psi}+C_{\phi} S_{\psi} & -C_{\theta} S_{\phi} S_{\psi}+C_{\phi} C_{\psi} & S_{\theta} S_{\phi} \\
-S_{\theta} C_{\psi} & S_{\theta} S_{\psi} & C_{\theta}
\end{array}\right] .}
\end{aligned}
$$

For the sake of simplicity, $\cos \theta$ and $\sin \theta$ are abbreviated as $C_{\theta}$ and $S_{\theta}$, respectively. Note that Eulerian angles depend on distance $u$. Let $\boldsymbol{\xi}, \boldsymbol{\eta}$, and $\boldsymbol{\zeta}$ be unit vectors along the $\xi_{-}, \eta$-, and $\zeta$-axes, respectively, at point $\mathrm{P}(u, 0)$. These unit vectors are given by the first, second, and third columns of the rotation matrix, respectively. Namely,

$$
A(\phi, \theta, \psi)=[\boldsymbol{\xi}|\boldsymbol{\eta}| \boldsymbol{\zeta}] .
$$

Let $\boldsymbol{x}(u, 0)=[x(u, 0), y(u, 0), z(u, 0)]^{\mathrm{T}}$ be the position vector of point $\mathrm{P}(u, 0)$. The position vector can be computed by integrating vector $\boldsymbol{\eta}(u, 0)$. Namely,

$$
\boldsymbol{x}(u, 0)=\boldsymbol{x}_{0}+\int_{0}^{u} \boldsymbol{\eta}(u, 0) \mathrm{d} u,
$$

where $\boldsymbol{x}_{0}=\left[x_{0}, y_{0}, z_{0}\right]^{\mathrm{T}}$ is the position vector at the end point $\mathrm{P}(0,0)$.

Let $\omega_{u \xi}, \omega_{u \eta}$, and $\omega_{u \zeta}$ be infinitesimal ratios of rotational angles around the $\xi$-, $\eta$-, and $\zeta$-axes, respectively, at point $\mathrm{P}(u, 0)$. They correspond to differentiation of rotational angles around these three axes with respect to distance $u$ and they are described as follows:

$$
\left[\begin{array}{c}
\omega_{u \xi} \\
\omega_{u \eta} \\
\omega_{u \zeta}
\end{array}\right]=\left[\begin{array}{c}
-S_{\theta} C_{\psi} \\
S_{\theta} S_{\psi} \\
C_{\theta}
\end{array}\right] \frac{\mathrm{d} \phi}{\mathrm{d} u}+\left[\begin{array}{c}
S_{\psi} \\
C_{\psi} \\
0
\end{array}\right] \frac{\mathrm{d} \theta}{\mathrm{d} u}+\left[\begin{array}{l}
0 \\
0 \\
1
\end{array}\right] \frac{\mathrm{d} \psi}{\mathrm{d} u} .
$$

Note that $\omega_{u \zeta}$ corresponds to bend of the object, $\omega_{u \eta}$ represents torsion of the object, and $\omega_{u \xi}$ indicates curvature of the central line in a longitudinal direction on the object.

\section{B. Description of Surface Bending}

Next, we consider general description of 3D surface. Let $\boldsymbol{x}(u, v)$ be the position vector of point $\mathrm{P}(u, v)$ on a surface. Let $\boldsymbol{x}_{u}(u, v)$ and $\boldsymbol{x}_{v}(u, v)$ be tangent vectors at point $\mathrm{P}(u, v)$ along $u$ - and $v$-axes, respectively, and let $e(u, v)$ be the normal vector at point $\mathrm{P}(u, v)$. According to differential geometry, the 
normal curvature $\kappa$ in direction $\boldsymbol{d}=a \boldsymbol{x}_{u}+b \boldsymbol{x}_{v}$ is represented as follows:

$$
\kappa=\frac{L a^{2}+2 M a b+N b^{2}}{E a^{2}+2 F a b+G b^{2}},
$$

where $E, F$, and $G$ are coefficients of the first fundamental form and $L, M$, and $N$ are those of the second fundamental form of the surface. These coefficients are defined as follows:

$$
\begin{aligned}
& E=\boldsymbol{x}_{u} \cdot \boldsymbol{x}_{u}, \\
& F=\boldsymbol{x}_{u} \cdot \boldsymbol{x}_{v}, \\
& G=\boldsymbol{x}_{v} \cdot \boldsymbol{x}_{v}, \\
& L=\frac{\partial \boldsymbol{x}_{u}}{\partial u} \cdot \boldsymbol{e}, \\
& M=\frac{\partial \boldsymbol{x}_{u}}{\partial v} \cdot \boldsymbol{e}, \\
& N=\frac{\partial \boldsymbol{x}_{v}}{\partial v} \cdot \boldsymbol{e} .
\end{aligned}
$$

The normal curvature $\kappa$ depends on the direction $\boldsymbol{d}$ and its maximum value $\kappa_{1}$ and its minimum value $\kappa_{2}$ are called the principal curvatures. Direction $\boldsymbol{d}_{1}$ of the maximum curvature $\kappa_{1}$ and direction $\boldsymbol{d}_{2}$ of the minimum curvature $\kappa_{2}$ are referred to as principal directions. The principal curvatures and the principal directions specify bend of a surface. A surface is also characterized by Gaussian curvature $K(u, v)$ and the mean curvature $H(u, v)$. They are related to the principal curvatures $\kappa_{1}$ and $\kappa_{2}$ by

$$
\begin{aligned}
& K=\kappa_{1} \kappa_{2}=\frac{L N-M^{2}}{E G-F^{2}}, \\
& H=\frac{\kappa_{1}+\kappa_{2}}{2}=\frac{E N-2 F M+G L}{2\left(E G-F^{2}\right)} .
\end{aligned}
$$

Vectors $\boldsymbol{x}_{u}, \boldsymbol{x}_{v}$, and $\boldsymbol{e}$ correspond to $\boldsymbol{\eta}, \boldsymbol{\zeta}$, and $\boldsymbol{\xi}$ in this paper, respectively. Then, coefficients of the first fundamental form are $E=1, F=0$, and $G=1$, respectively. Moreover, the derivation of unit vectors $\boldsymbol{\eta}$ and $\zeta$ can be described using infinitesimal ratios of rotational angles as follows:

$$
\begin{aligned}
& \frac{\partial \boldsymbol{\eta}}{\partial u}=-\omega_{u \zeta} \boldsymbol{\xi}+\omega_{u \xi} \boldsymbol{\zeta}, \\
& \frac{\partial \boldsymbol{\zeta}}{\partial u}=\omega_{u \eta} \boldsymbol{\xi}-\omega_{u \xi} \boldsymbol{\eta}=\frac{\partial \boldsymbol{\xi}}{\partial v} .
\end{aligned}
$$

Substituting eqs.(14) and (15) into eqs.(9) and (10), $L$ and $M$ can be represented as a function of infinitesimal angle ratios as follows:

$$
\begin{aligned}
& L=\left(-\omega_{u \zeta} \boldsymbol{\xi}+\omega_{u \xi} \boldsymbol{\zeta}\right) \cdot \boldsymbol{\xi}=-\omega_{u \zeta}, \\
& M=\left(\omega_{u \eta} \boldsymbol{\xi}-\omega_{u \xi} \boldsymbol{\eta}\right) \cdot \boldsymbol{\xi}=\omega_{u \eta} .
\end{aligned}
$$

In contrast, $N$ cannot be described by Eulerian angles. So, we introduce the fourth parameter $\delta(u, 0): N=\delta(u, 0)$. It corresponds to the curvature in a transverse direction. Consequently, Gaussian curvature $K$ and the mean curvature $H$ is described by

$$
\begin{aligned}
& K=-\omega_{u \zeta} \delta-\omega_{u \eta}^{2}, \\
& H=\frac{-\omega_{u \zeta}+\delta}{2} .
\end{aligned}
$$

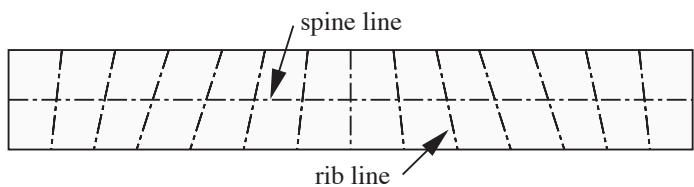

Fig. 3. Fishbone model

Thus, bending of a surface is characterized by Eulerian angles $\phi(u, 0), \theta(u, 0)$, and $\psi(u, 0)$ and the curvature in a transverse direction $\delta(u, 0)$. Note that $K$ and $H$ depends on not only coordinate $u$ but also coordinate $v$. In this paper, we assume that the whole shape of a belt object can be described by the shape of the central axis in a longitudinal direction because the width of a belt object is sufficiently small compared to its length.

If a principal curvature $\kappa_{2}$, i.e., the minimum value of the normal curvature is equal to zero, the surface is developable. Namely, it can be flattened without its expansion or contraction. Such surface is referred to as a developable surface. In this paper, we assume that a belt object is inextensible. Then, the deformed shape of the object corresponds to a developable surface. It means that the object bends in direction $\boldsymbol{d}_{1}$ and it is not deformed in direction $\boldsymbol{d}_{2}$. Namely, a line the direction of which coincides with direction $\boldsymbol{d}_{2}$ is kept straight after deformation. In this paper, the central axis in a longitudinal direction of the object is referred to as the spine line and a line with zero curvature at a point on the object is referred to as a rib line as shown in Fig.3. We assume that bend and torsion of the spine line and direction of the rib line of each point specifies deformation of a belt object. This model is referred to as a fishbone model in this paper. Let $\alpha(u, 0)$ be rib angle, which is the angle between the spine line and direction $\boldsymbol{d}_{1}$ as shown in Fig.4-(a). Let $\boldsymbol{r}(u)$ be a unit vector along a rib line at point $\mathrm{P}(u, 0)$ on the spine line. It is described by

$$
\boldsymbol{r}=-\boldsymbol{\eta} \sin \alpha+\boldsymbol{\zeta} \cos \alpha .
$$

Then, coordinates of a point on a rib line and on either longitudinal edge $\boldsymbol{x}\left(u^{\prime}, \pm V / 2\right)$ is represented as follows:

$$
\boldsymbol{x}\left(u^{\prime}, \pm V / 2\right)=\boldsymbol{x}(u, 0) \pm \frac{V}{2 \cos \alpha(u, 0)} \boldsymbol{r}(u, 0),
$$

where $u^{\prime}$ satisfies

$$
u^{\prime}=u+\frac{V}{2} \tan \alpha(u, 0) .
$$

Consequently, the whole shape of a belt object can be represented using five variables $\phi(u), \theta(u), \psi(u), \delta(u)$, and $\alpha(u)$. Note that they depend on only the distance $u$ from one end of the object along the spine line.

\section{Constraints on Belt Object Variables}

Let us consider conditions which five variables must satisfy so that the surface of a belt object is developable. Gaussian curvature $K$ of a developable surface must be zero at any point. So, the following constraint is imposed on the object.

$$
K=-\omega_{u \zeta} \delta-\omega_{u \eta}^{2}=0, \quad \forall u \in[0, U] .
$$




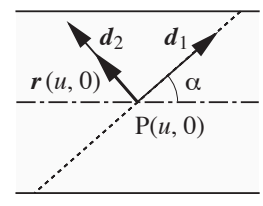

(a)

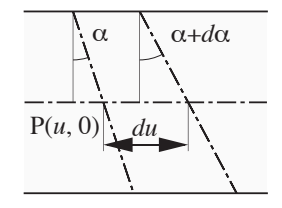

(b)
Fig. 4. Rib angle and rib lines

From eq.(23), $\delta$ is described by

$$
\delta=-\frac{\omega_{u \eta}^{2}}{\omega_{u \zeta}}
$$

Recall that infinitesimal ratio of rotational angle around $\xi$ axis $\omega_{u \xi}$ indicates curvature of the spine line on the object. In the initial state, the spine line is straight, that is, its curvature is constantly equal to zero. So, $\omega_{u \xi}$ must be satisfied the following equation after any deformation because of the inextensibility of a belt object:

$$
\omega_{u \xi}=0, \quad \forall u \in[0, U]
$$

Moreover, as shown in Fig.4-(b), to prevent rib lines from intersecting with themselves on a belt object, the following inequalities must be satisfied:

$$
\begin{aligned}
& \frac{V}{2} \tan \alpha+\mathrm{d} u \geq \frac{V}{2} \tan (\alpha+\mathrm{d} \alpha), \\
& \frac{V}{2} \tan (\alpha+\mathrm{d} \alpha)+d u \geq \frac{V}{2} \tan \alpha .
\end{aligned}
$$

Then, rib angle $\alpha$ at any point on the spine line must be satisfied

$$
-\frac{2 \cos ^{2} \alpha}{V} \leq \frac{\mathrm{d} \alpha}{\mathrm{d} u} \leq \frac{2 \cos ^{2} \alpha}{V}, \forall u \in[0, U] .
$$

Substituting eq.(24) into eqs.(5) and (19), The normal curvature in direction $\boldsymbol{d}_{1}=\boldsymbol{\xi} \cos \alpha+\boldsymbol{\eta} \sin \alpha$, i.e., a principal curvature $\kappa_{1}$ is as follows:

$$
\begin{aligned}
\kappa_{1} & =-\omega_{u \zeta} \cos ^{2} \alpha+2 \omega_{u \eta} \cos \alpha \sin \alpha-\frac{\omega_{u \eta}^{2}}{\omega_{u \zeta}} \sin ^{2} \alpha \\
& =-\omega_{u \zeta}-\frac{\omega_{u \eta}^{2}}{\omega_{u \zeta}}
\end{aligned}
$$

Then, $\alpha$ can be described as follows:

$$
\alpha=-\tan ^{-1} \frac{\omega_{u \eta}}{\omega_{u \zeta}} .
$$

Now, let us introduce parameter $\beta(u)$ :

$$
\beta=\tan \alpha
$$

Then, $\beta$ must satisfy the following equation from eq.(30):

$$
\omega_{u \eta}+\omega_{u \zeta} \beta=0, \forall u \in[0, U] .
$$

Moreover, eq.(28) is described as follows by substituting eq.(31):

$$
-\frac{2}{V} \leq \frac{\mathrm{d} \beta}{\mathrm{d} u} \leq \frac{2}{V}, \forall u \in[0, U]
$$

Consequently, the shape of a belt object can be represented by four functions $\phi(u), \theta(u), \psi(u)$, and $\beta(u)$. And, they must satisfy eqs.(25), (32), and (33) in any state to maintain developability.

\section{Potential Energy and Geometric Constraints}

Let us formulate the potential energy of a deformed belt object. We can assume that a belt object bends along direction $\boldsymbol{d}_{1}$ without torsional deformation. This implies that the shape of cross-section along rib line is fixed while that along a transverse direction can change. Then, the potential energy $I$ can be described as follows assuming that the flexural energy is proportional to the bending moment at each point $\mathrm{P}(u)$ :

$$
I=\int_{0}^{U} \frac{R_{f}}{2 \cos \alpha} \kappa_{1}^{2} \mathrm{~d} u=\int_{0}^{U} \frac{R_{f}}{2 \cos \alpha} \frac{\left(\omega_{u \zeta}^{2}+\omega_{u \eta}^{2}\right)^{2}}{\omega_{u \zeta}^{2}} \mathrm{~d} u,
$$

where $R_{f}$ represents the flexural rigidity of a belt object along the spine line at point $\mathrm{P}(u)$. If rib angle $\alpha$ is equal to zero, the width of an infinitesimal region for integration coincides with object width $V$. Then, $R_{f} / \cos \alpha$ corresponds to the flexural rigidity along the spine line. If $\alpha$ is not equal to zero, the width of a infinitesimal region becomes longer than the object width. This means that the latter region has larger potential energy than the former region even if they have the same principal curvature $\kappa_{1}$.

Next, let us formulate geometric constraints imposed on a belt object. The relative position between two points on the spine line of the object is often controlled during a manipulative operation of the object. Consider a constraint that specifies the positional relationship between two points on the object. Let $\boldsymbol{l}=\left[l_{x}, l_{y}, l_{z}\right]^{T}$ be a predetermined vector describing the relative position between two operational points on the spine line, $\mathrm{P}\left(u_{a}\right)$ and $\mathrm{P}\left(u_{b}\right)$. Recall that the spatial coordinates corresponding to distance $u$ are given by eq.(3). Thus, the following equation must be satisfied:

$$
\boldsymbol{x}\left(u_{b}\right)-\boldsymbol{x}\left(u_{a}\right)=\boldsymbol{l}
$$

The orientation at one point on the spine line of the object is often controlled during an operation as well. This constraint is simply described as follows:

$$
A\left(\phi\left(u_{c}\right), \theta\left(u_{c}\right), \psi\left(u_{c}\right)\right)=A\left(\phi_{c}, \theta_{c}, \psi_{c}\right),
$$

where $\phi_{c}, \theta_{c}$, and $\psi_{c}$ are predefined Eulerian angles at one operational point $\mathrm{P}\left(u_{c}\right)$.

Therefore, the shape of a belt object is determined by minimizing the potential energy described by eq.(34) under necessary constraints for developability described by eqs.(25), (32), and (33) and geometric constraints imposed on the object described by eqs.(35) and (36). Namely, computation of the deformed shape of the object results in a variational problem under equational and inequality constraints.

\section{Computation of Belt Object Deformation}

\section{A. Computation Algorithm}

Computation of the deformed shape of a belt object results in a variational problem as mentioned in the previous section. 
In [12], we developed an algorithm based on Ritz's method[13] and a nonlinear programming technique to compute linear object deformation. In this paper, we apply such algorithm to the computation of belt object deformation.

Let us express functions $\phi(u), \theta(u), \psi(u)$, and $\beta(u)$ by linear combinations of basic functions $e_{1}(u)$ through $e_{n}(u)$ :

$$
\begin{aligned}
& \phi(u)=\sum_{i=1}^{n} a_{i}^{\phi} e_{i}(u) \triangleq \boldsymbol{a}^{\phi} \cdot \boldsymbol{e}(u), \\
& \theta(u)=\sum_{i=1}^{n} a_{i}^{\theta} e_{i}(u) \triangleq \boldsymbol{a}^{\theta} \cdot \boldsymbol{e}(u), \\
& \psi(u)=\sum_{i=1}^{n} a_{i}^{\psi} e_{i}(u) \triangleq \boldsymbol{a}^{\psi} \cdot \boldsymbol{e}(u), \\
& \beta(u)=\sum_{i=1}^{n} a_{i}^{\beta} e_{i}(u) \triangleq \boldsymbol{a}^{\beta} \cdot \boldsymbol{e}(u),
\end{aligned}
$$

where $\boldsymbol{a}^{\phi}, \boldsymbol{a}^{\theta}, \boldsymbol{a}^{\psi}$, and $\boldsymbol{a}^{\beta}$ are vectors consisting of coefficients corresponding to functions $\phi(u), \theta(u), \psi(u)$, and $\beta(u)$ respectively, and vector $\boldsymbol{e}(u)$ is composed of basic functions $e_{1}(u)$ through $e_{n}(u)$. Substituting the above equations into eq.(34), potential energy $I$ is described by a function of coefficient vectors $\boldsymbol{a}^{\phi}, \boldsymbol{a}^{\theta}, \boldsymbol{a}^{\psi}$, and $\boldsymbol{a}^{\beta}$. Constraints are also described by conditions involving the coefficient vectors. Especially, discretizing eqs.(25), (32), and (33) by dividing interval $[0, U]$ into $n$ small intervals yields a finite number of conditions. As a result, a set of the constraints is expressed by equations and inequalities in terms of the coefficient vectors.

Consequently, the deformed shape of a belt object can be derived by computing a set of coefficient vectors $\boldsymbol{a}^{\phi}$, $\boldsymbol{a}^{\theta}, \boldsymbol{a}^{\psi}$, and $\boldsymbol{a}^{\beta}$ that minimizes the potential energy under the constraints. This minimization problem can be solved by the use of a nonlinear programming technique such as the multiplier method[14]. In this method, Lagrange multipliers are introduced as variables for optimization to satisfy given constraints.

\section{B. Examples of Computation}

In this section, numerical examples demonstrate how the proposed method computes the deformed shape of a belt object. The following set of basic functions are used in the computation of these examples:

$$
\begin{aligned}
& e_{1}=1, \quad e_{2}=u, \\
& e_{2 i+1}=\sin \frac{\pi i u}{U}, \\
& e_{2 i+2}=\cos \frac{\pi i u}{U}, \quad(i=1,2,3,4) .
\end{aligned}
$$

Assume that the length of the object $U$ is equal to 1 , its width $V$ is equal to 0.1 , and its flexural rigidity along the spine line $R_{f}$ is constantly equal to 1 . Necessary constraints for developability described by eqs.(25), (32), and (33) are divided into 16 conditions at point $\mathrm{P}(i U / 15)(i=0, \cdots, 15)$ respectively in the following examples. All computations were performed on a $750 \mathrm{MHz}$ Alpha $21264 \mathrm{CPU}$ with $512 \mathrm{MB}$

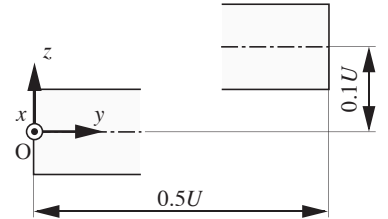

Fig. 5. Example

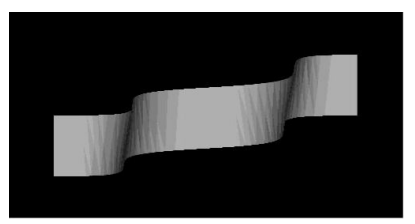

(a) Top view

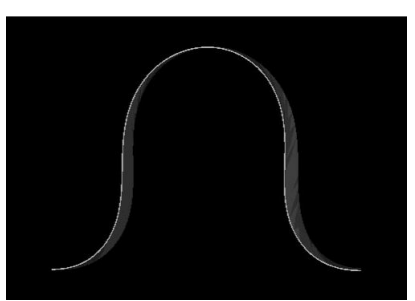

(b) Front view

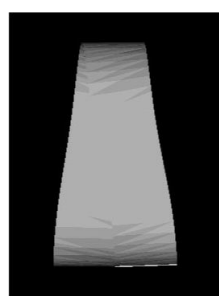

(c) Side view
Fig. 6. Computational result of example 1

memory operated by Tru64UNIX. Programs were compiled by a Compaq C Compiler V6.1 with optimization option -O4.

Fig.5 shows the first example of belt object deformation. In this example, positional constraints imposed on a belt object are described by

$$
\boldsymbol{x}(U)=\int_{0}^{U} \boldsymbol{\eta}(u) \mathrm{d} u=\left[\begin{array}{c}
0 \\
0.5 \\
0.1
\end{array}\right] U .
$$

Orientational constraints are represented as follows:

$$
\begin{gathered}
\phi(0)=\theta(0)=\psi(0)=\beta(0)=0, \\
\phi(U)=\theta(U)=\psi(U)=\beta(U)=0 .
\end{gathered}
$$

This means that directions of the spine line at both ends are parallel but they are not collinear. Then, this optimization problem has 40 variables for Eulerian angles and the rib angle, 11 for geometrical constraints, and 64 for necessary constraints for developability. Fig.6 shows computational results. Fig.6(a), -(b), and -(c) illustrate the top, front, and side view of the object, respectively. As shown in this figure, the object is bent and twisted to satisfy given geometric constraints. This implies that rib angle $\alpha$ varies with distance $u$. Fig.7 shows the relationship between $\alpha$ and $u$. Considering eq.(34), it is found that $\alpha$ becomes smaller, that is, $\cos \alpha$ becomes larger at a point with a large curvature such as the midpoint of the object to reduce its potential energy. The maximum height of the object is $0.35 \mathrm{U}$. The computation time was about 1200 seconds.

Fig.8 shows the second example. Positional and orienta- 


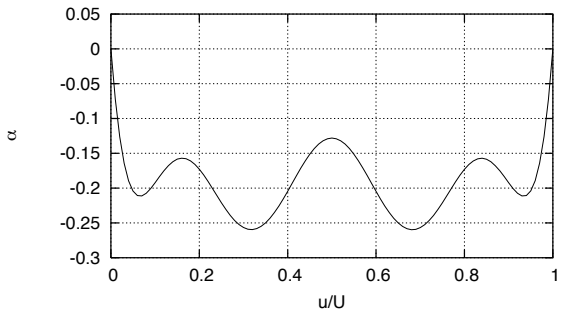

Fig. 7. Rib angle in example 1

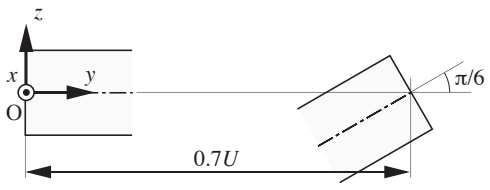

Fig. 8. Example 2

tional constraints are described by

$$
\begin{gathered}
\boldsymbol{x}(U)=\int_{0}^{U} \boldsymbol{\eta}(u) \mathrm{d} u=\left[\begin{array}{c}
0 \\
0.7 \\
0
\end{array}\right] U \\
\phi(0)=\theta(0)=\psi(0)=\beta(0)=0 \\
\boldsymbol{\eta}(U)=\left[\begin{array}{c}
0 \\
\cos (\pi / 6) \\
\sin (\pi / 6)
\end{array}\right], \boldsymbol{\zeta}(U)=\left[\begin{array}{c}
0 \\
-\sin (\pi / 6) \\
\cos (\pi / 6)
\end{array}\right], \\
\beta(U)=0 .
\end{gathered}
$$

Namely, both end of the spine line are on the same line but directions of the spine line at these points are different. Fig.9 shows computational results and Fig.10 shows the relationship between $\alpha$ and $u$. As shown in these figures, at parts close to both ends of the object, where the object kinks, $\alpha$ has a large value. Coordinates of the object peak are $[0.3 U, 0.4 U,-0.01 U]^{T}$. This computation took about 1500 seconds.

\section{EXPERIMENTS OF Belt OBJeCt Deformation}

In this section, the computation results are experimentally verified by measuring the deformed shape of a belt object. We

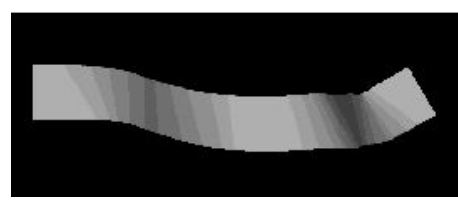

(a) Top view
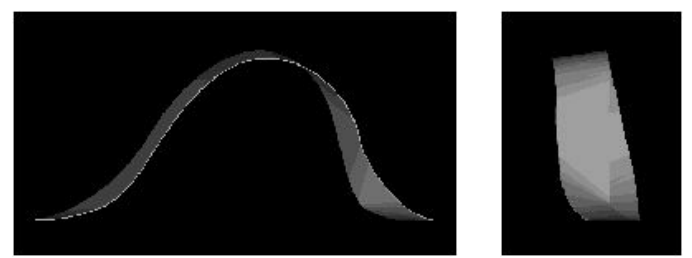

(b) Front view

(c) Side view

Fig. 9. Computational result of example 2

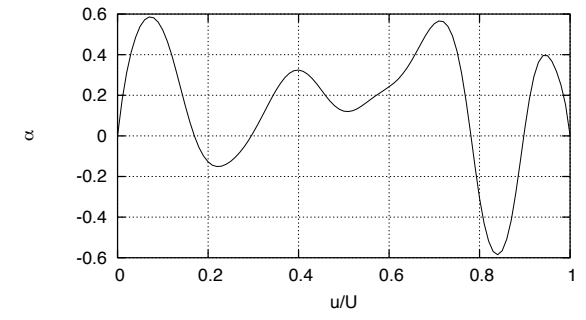

Fig. 10. Rib angle in example 2

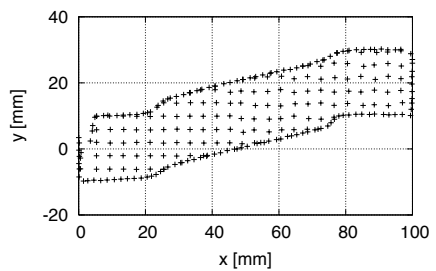

(a) Top view

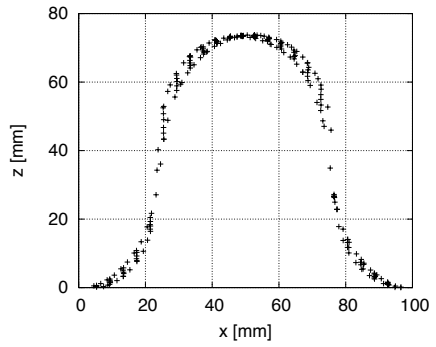

(b) Front view

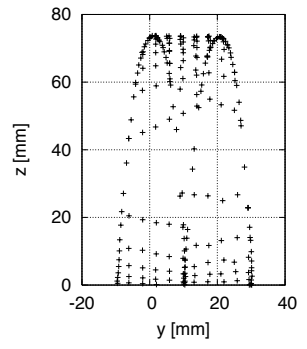

(c) Side view
Fig. 11. Experimental result of example 1

measured the shape of a rectangular polystyrol sheet which is $200 \mathrm{~mm}$ long, $20 \mathrm{~mm}$ wide, and $140 \mu \mathrm{m}$ thick with a 3D scanner. Their flexural rigidity is unknown but from eq.(34), it is found that the deformed shape is independent of it when it is constant along the spine line. Fig.11 shows the experimental result of deformation illustrated in Fig.5. The computational result shown in Fig.6 almost coincide with this experimental result. Fig. 12 shows the experimental result of deformation illustrated in Fig.8. As shown in this figure, the computed shape on $x y$ and $x z$-planes is qualitatively similar to the actual shape and $x$ and $y$-coordinates of the object peak almost coincide. Thus, our method can estimate bending and torsional deformation of a rectangular belt object using only flexural rigidity of the object along its spine line if the object is isotropic.

\section{Discussion of Fishbone Model}

In this section, we discuss our proposed model. Recall that the surface of an inextensible belt object corresponds to a developable surface, which is a kind of ruled surfaces. A ruled surface is a surface that can be swept out by moving a straight line, which is called a ruling, in 3D space and it can be formulated as follows:

$$
\boldsymbol{x}(u, v)=\boldsymbol{p}(u)+v \boldsymbol{q}(u),
$$

where $\boldsymbol{p}(u)$ and $\boldsymbol{q}(u)$ are referred to as the base curve and the director curve, respectively. Rib lines in the fishbone model 


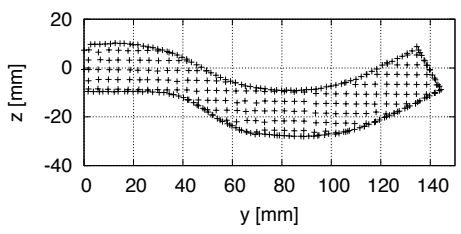

(a) Top view

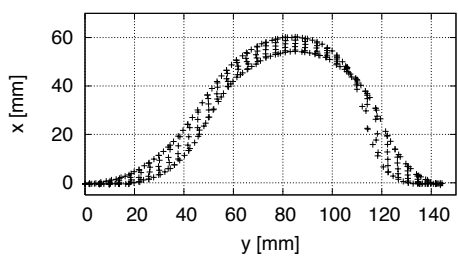

(b) Front view

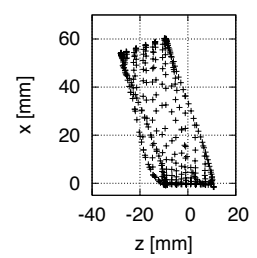

(c) Side view
Fig. 12. Experimental result of example 2

correspond to rulings. Moreover, $\boldsymbol{x}$ and $\boldsymbol{r}$ in eq.(21) are similar to the base curve and the director curve, respectively. The formulation described by eq.(51) is sufficient to represent the object surface after deformation. However, it is not suitable for representation of energy increment from the initial shape. To estimate potential energy of the object and to derive its stable shape, we have to specify dependent parameters on deformation and independent parameters of deformation. As a belt object is assumed to be inextensible, its shape in $u v$ space is not changed by any deformation. This means that the length, width, and angle between $u$ - and $v$-axes are constant. So, $E=1, G=0, F=1$. Furthermore, the constraint described by eq.(25) is added for straightness of the spine line in $u v$-space. Then, the object only can be bent around $\zeta$ axis and twisted around $\eta$-axis, and the rib angle is determined from these bend and torsion. As mentioned before, the object shape is represented by four variables $\phi(u), \theta(u), \psi(u)$, and $\beta(u)$. Note that they must satisfy constraints described by eqs.(25) and (32). Therefore, we can conclude that deformation of an inextensible belt object is described by two independent variables.

Some flexible circuit boards bend like a polygonal line as shown in Fig.1 or curve like a circular arc. Let us discuss application of our model to such bent/curved boards. First, to represent a belt object with multiple bends, Eulerian angles and rib angles of straight parts between bends should be defined separately. The deformed shape of the object is then derived by minimizing total potential energy of each part. But, continuity of the rib line at each bend should be discussed. Fig.14 shows a computational result of deformation of a belt object with one bend illustrated in Fig.13. Next, let us consider a curved belt object. As we assume that the spine line is straight in this paper, $\omega_{u \xi}$ is constantly equal to zero. If an object is curved with a certain curvature, $\omega_{u \xi}$ must be equal to that curvature even if the object deforms. We can impose this constraint on the object instead of eq.(25). This implies that our proposed method can be applied to a curved belt object. Fig.16 shows a computational result of deformation of a curved belt object illustrated in Fig.15. Thus, our proposed

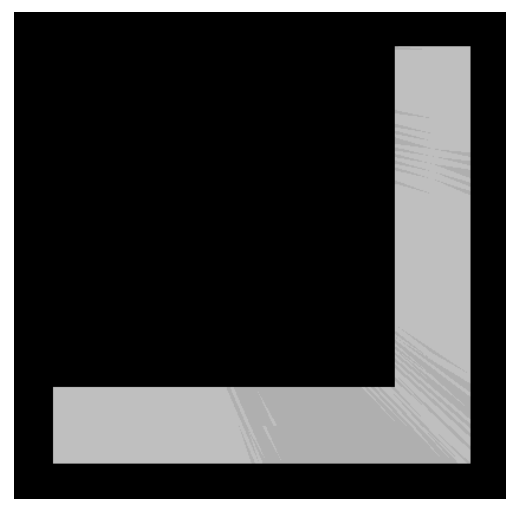

Fig. 13. Bent Belt Object

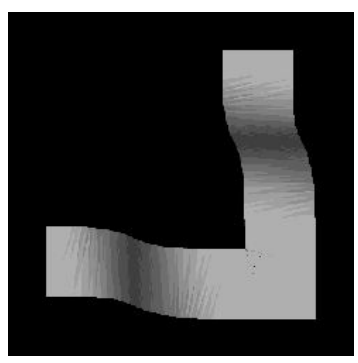

(a) Top view

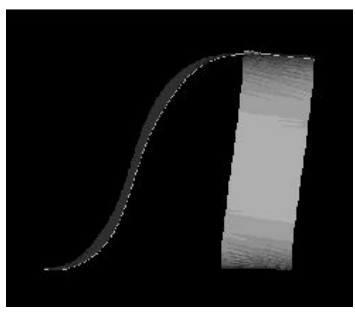

(b) Front view

$\begin{array}{ll}\text { (c) Side view } & \text { Front view }\end{array}$

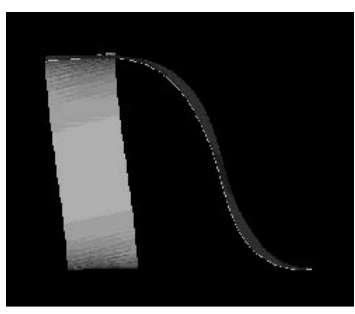

method can represent deformation of various belt objects.

\section{CONCLUSIONS}

A fishbone model based on differential geometry to represent belt object deformation was proposed toward manipulation/assembly of film/flexible circuit boards. First, deformation of a rectangular belt object was described using differential geometry. Next, the fishbone model was proposed by considering characteristics of a developable surface. In this model, deformation of a belt object is represented using the shape of the spine line and the direction of straight rib lines. Then, we can estimate belt object deformation if only the flexural rigidity of the object along the spine line is given. After that, we formulate potential energy of the object and constraints imposed on it. Finally, a procedure to compute the deformed shape of the object was explained and some computational results were compared with experimental results. They demonstrated that the fishbone model can represent deformation of a belt object qualitatively well. 


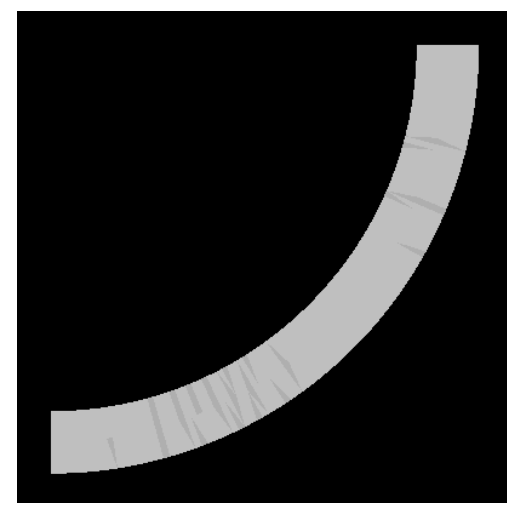

Fig. 15. Curved Belt Object

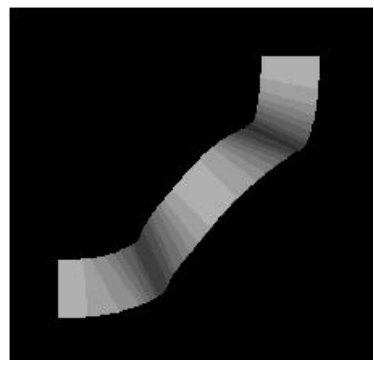

(a) Top view

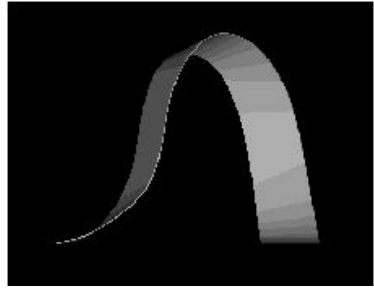

(b) Front view

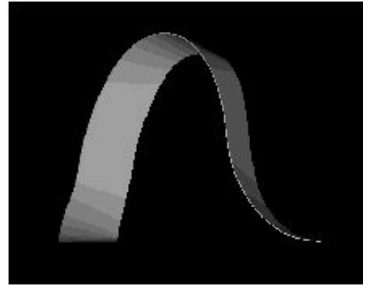

Fig. 16. Deformation of Curved Belt Object

\section{REFERENCES}

[1] S. Timoshenko, "Theory of Plates and Shells", McGraw-Hill Book Company, Inc., 1940.

[2] E. H. Mansfield, "The Inextensional Theory for Thin Flat Plates", The Quarterly J. Mechanics and Applied Mathematics, Vol.8, pp.338-352, 1955.

[3] A. Witkin and W. Welch, "Fast Animation and Control of Nonrigid Structures", Computer Graphics, Vol.24, pp.243-252, 1990.

[4] D. K. Pai, "STRANDS: Interactive Simulation of Thin Solids using Cosserat Models", Computer Graphics Forum, Vol.21, No.3, pp.347-352, 2002.

[5] Y. E. Zheng, R. Pei, and C. Chen, "Strategies for Automatic Assembly of Deformable Objects", Proc. of IEEE Int. Conf. Robotics and Automation, pp.2598-2603, 1991.

[6] H. Nakagaki, K. Kitagaki, and H. Tsukune, "Study of Insertion Task of a Flexible Beam into a Hole", Proc. of IEEE Int. Conf. Robotics and Automation, pp.330-335, 1995.

[7] K. Kosuge, M. Sasaki, K. Kanitani, H. Yoshida, and T. Fukuda, "Manipulation of a Flexible Object by Dual Manipulators", Proc. of Int. Conf. Robotics and Automation, pp.318-323, 1995

[8] F. Lamiraux and L. E. Kavraki, "Planning Paths for Elastic Objects under Manipulation Constraints", Int. J. Robotics Research, Vol.20, No.3, March, pp.188-208, 2001.

[9] D. Sun, J. K. Mills, and Y. Liu, "Position Control of Robot Manipulators Manipulating a Flexible Payload", Int. J. Robotics Research, Vol.18, No.3, March, pp.319-332, 1999.

[10] A. Gray, "Modern Differential Geometry of Curves and Surfaces", CRC Press, 1993.

[11] M. Moll and L. E. Kavraki, "Path Planning for Variable Resolution Minimal-Energy Curves of Constant Length", Proc. IEEE Int. Conf. Robotics and Automation, pp.2142-2147, 2005.

[12] H. Wakamatsu and S. Hirai, "Static Modeling of Linear Object Deformation Based on Differential Geometry", Int. J. Robotics Research, Vol.23, No.3, pp.293-311, 2004.

[13] L. E. Elsgolc, "Calculus of Variations", Pergamon Press, 1961.

[14] M. Avriel, "Nonlinear Programming: Analysis and Methods", PrenticeHall, 1976. 